\title{
Notas taxonômicas sobre Exaerete (Hymenoptera: Apidae: Euglossina), com a descrição de uma nova espécie
}

\author{
Marcio Luiz de Oliveira ${ }^{1,2}$ \\ ${ }^{1}$ Instituto Nacional de Pesquisas da Amazônia, Coordenação de Pesquisas em Entomologia, \\ Av. André Araújo, n. 2936, Petrópolis, CP 478, CEP 69011-970, Manaus, AM, Brasil \\ ${ }^{2}$ Autor para correspondência: Marcio Luiz,de Oliveira, e-mail: mlolivei@inpa.gov.br
}

OLIVEIRA, M.L. Taxonomic notes on Exaerete (Hymenoptera: Apidae: Euglossina), with the description of a new species. Biota Neotrop. 11(1): http://www.biotaneotropica.org.br/v11n1/en/abstract?article+bn02011012011.

Abstract: Exaerete kimseyae sp. n., a cleptoparasitic bee apparently endemic to Panama is described. This new species was misidentified by Kimsey (1979) as E. trochanterica (Friese, 1910). For this reason Anjos-Silva \& Rebêlo (2006), not knowing the true identity of Exaerete trochanterica, described an individual of this species collected in Mato Grosso, Brazil as a new taxon, Exaerete guaycuru. Here E. guaycuru is placed as junior synonym of Exaerete trochanterica.

Keywords: orchid bees, parasitic bees, Panama, synonymy.

OLIVEIRA, M.L. Notas taxonômicas sobre Exaerete (Hymenoptera: Apidae: Euglossina), com a descrição de uma nova espécie. Biota Neotrop. 11(1): http://www.biotaneotropica.org.br/v11n1/pt/ abstract?article+bn02011012011.

Resumo: Exaerete kimseyae sp. n., abelha de hábito cleptoparasítico e provavelmente endêmica do Panam , é aqui descrita. Esta nova espécie foi erroneamente identificada como E. trochanterica (Friese, 1910) por Kimsey (1979). Por esse motivo, Anjos-Silva \& Rebêlo (2006), desconhecendo a correta identidade de Exaerete trochanterica, descreveram um exemplar desta espécie coletado em Mato Grosso, Brasil como Exaerete guaycuru. Aqui E. guaycuru é colocada como sinônimo júnior de Exaerete trochanterica.

Palavras-chave: abelhas das orquídeas, abelhas parasitas, Panamá, sinonímia. 


\section{Introdução}

O gênero Exaerete Hoffmannsegg, 1817, pertencente à subtribo Euglossina, é tipicamente neotropical e constituído de abelhas de coloração verde-metálica, cujo tamanho corporal varia de 15 a $28 \mathrm{~mm}$. As fêmeas são cleptoparasíticas e colocam seus ovos exclusivamente em ninhos de Eulaema Lepeletier, 1841 e Eufriesea Cockerell, 1899, dois outros gêneros de Euglossina (Garófalo \& Rozen Jr. 2001). Exaerete tem distribuição do México até a Argentina (Moure 1964, Kimsey 1979, Michener 2000, Oliveira \& Nemésio 2003, Roubik \& Hanson 2004, Anjos-Silva \& Rebêlo 2006).

Segundo Kimsey (1979), E. trochanterica ocorre do Panamá até o Norte do Brasil, entretanto, o único exemplar conhecido do Panamá, o mesmo que aparece no trabalho de Kimsey (1979), foi aqui examinado e difere substancialmente do tipo e de dezenas de exemplares examinados de E. trochanterica, o que permite descrevê-lo como uma nova espécie.

\section{Material e Métodos}

Foram examinados o tipo de E. trochanterica depositado no Museu de Berlim, um parátipo de E. guaycuru emprestado por E. J. Anjos-Silva e dezenas de exemplares das outras espécies do gênero, com exceção de E. azteca Moure, 1964. Com relação à pontuação do tegumento, foram considerados: 1) padrão de distribuição: regular ou irregular; 2) densidade: densa (intervalos entre os pontos menores que os diâmetros dos pontos) ou esparsa (intervalos maiores que os diâmetros dos pontos); 3) forma dos pontos: circular ou biselada; e 4) tamanho dos pontos, vistos com objetiva de $0,63 \times$ : pequena (menor ou igual $0,15 \mathrm{~mm}$ ); média (entre 0,16 e 0,32 ) e grande (igual ou maior que 0,33 ).

\section{Resultados}

\section{EXAERETE KIMSEYAE SP. N.}

Exaerete trochanterica; Kimsey, 1979. J. Kansas Ent. Soc., 52(4): 736-740, 742, 744-746 (Tax., Ch., Figs., Dist. Geo., Redescr., partim).

Material Tipo: Holótipo, macho, com os seguintes dados "Canal Zone, Pan [sic], Barro Colorado I. [sic], VIII.15.1977 [data manuscrita à tinta], RB \& LS Kimsey, Exaerate [sic] trochanterica (Friese), ô, det. LS Kimsey" [esternos VII, VIII e cápsula genital colados em etiqueta adicional] [depositado na coleção da Universidade da Califórnia em Davis]. Coordenadas geográficas da localidade-tipo: $09^{\circ} 09^{\prime} \mathrm{N}$ e $79^{\circ} 50^{\prime} \mathrm{W}$.

Diagnose: Semelhante a $E$. trochanterica mas diferindo pela área lisa nas laterais das tégulas, próximo aos escleritos alares; no escutelo com margem posterior reta e truncada (Figura 3 ), com área central relativamente desprovida de pontos. $\mathrm{O}$ bordo posterior dos tergos II a VI com área lisa tão longa quanto o diâmetro do ocelo médio (área lisa mais estreita em E. trochanterica). A pilosidade é escura e longa nas genas (Figura 2) (clara e mais curta em E. trochanterica). O esterno VII é um arco aberto, com profunda reentrância longitudinal mediana (Figura 5) (ver também figura 24a de Kimsey, 1979). Nisso, difere completamente de E. trochanterica, conforme se pode ver nas figuras 22a e 23a de Anjos-Silva \& Rebêlo (2006).

Fêmea: desconhecida.

Iscas: desconhecidas.

Etimologia: Homenagem a Lynn Siri Kimsey, coletora do holótipo.

Descrição:

Coloração. Corpo verde-metálico no geral, mas com reflexos dourados no ápice do clípeo, disco do mesoscuto, propódeo, tergos I a V e tíbias posteriores; verde-metálico com reflexos violáceos na face anterior das pernas; membrana das asas castanho-escuro.

Pilosidade. Esparsa, relativamente curta e com pêlos claros mesclados com escuros na face e disco do mesoscuto; semelhante, porém com pêlos mais longos nos mesepisternos e lobos pronotais; menos esparsa, longa e predominantemente escura nas genas (Figura 2), face posterior das pernas dianteiras, tíbias médias e franja pósglandular ; mais densa, clara e moderadamente longa no propódeo, metepisternos, cantos inferiores do escutelo, trcnteres dianteiros, coxas traseiras e junto à fenda esternal; densa, escura e curta na face interna dos tarsos médios e traseiros.

Pontuação. Mal definida e densa no labro; regular, relativamente densa, circular aberta inferiormente e média ( 2 traços) na porção inferior do clípeo, esparsa na porção superior e áreas para-oculares inferiores; irregular, esparsa, circular e pequena (1 traço) na base das mandíbulas; regular, esparsa e circular aberta inferiormente nas áreas para-oculares superiores; regular, densa, circular e média no entorno dos alvéolos; irregular, densa, circular e pequena junto à base da linha frontal; regular, densa, biselada e média na área ante-ocelar; irregular; esparsa, circular, de pequena a média no vértice, porém menos densa nas genas; irregular, esparsa, circular e de pequena a média, porém rasa, nos lobos pronotais e metepisternos; regular, esparsa, circular e de pequena a média no mesoscuto; regular, densa, circular, pequena e rasa nas tégulas, com pequena porção lisa nas laterais, próximo aos escleritos alares; regular, densa, circular nos mesepisternos; irregular, esparsa, circular, de média a grande no escutelo, mas com área relativamente lisa no centro; irregular, bastante esparsa, circular, pequena e rasa na face externa das coxas e fêmures posteriores; irregular na face externa das tíbias posteriores, mal definida e densa na porção proximal, bastante esparsa, circular, média e rasa na distal; regular, densíssima, circular e pequena nos tergos I a VI, mas com o bordo posterior liso e equivalente ao diâmetro do ocelo médio na região mais central dos tergos II a VI, depois alargando-se para as laterais; mais densa ainda e pequena no centro dos tergos V e VI; irregular, esparsa, circular e pequena no esterno II, aumentando em densidade do III ao V, praticamente inexistente no VI.

Mensurações e Estrutura. Comprimento total $23 \mathrm{~mm}$; largura da cabeça $6,5 \mathrm{~mm}$, comprimento $5,7 \mathrm{~mm}$, distância interorbital superior 2,8 mm; comprimento dos escapos 2,2 $\mathrm{mm}$; comprimento dos olhos 4,2 mm; língua 12,7 mm, em repouso alcança o esterno I; palpos labiais tetra-segmentados; fronte sem tubérculo mediano (Figura 1). Largura do mesossoma $8 \mathrm{~mm}$; comprimento das asas anteriores $24 \mathrm{~mm}$; largura do escutelo $4.6 \mathrm{~mm}$, comprimento $2.3 \mathrm{~mm}$, com dois tubérculos laterais; margem escutelar posterior reta (Figura 3); hipo-epímero sem tubérculo; largura do abdomen $7.6 \mathrm{~mm}$. Área "aveludada” cobrindo grande parte das tíbia médias, exceto em uma estreita faixa anterior que se alarga na porção distal; coxim longo e triangular, seus lados aproximadamente o triplo da base. Tíbia posterior com fenda glandular atingindo o ápice; na porção distal da face anterior, duas projeções dentiformes de onde saem os esporões (Figura 4). Esterno VII em arco aberto e com profunda reentrância longitudinal mediana (Figura 5), com um conjunto de cerdas apicais e fileiras de cerdas mais curtas centralmente; esterno VIII triangular apicalmente, o ápice mais estreitado junto aos pequenos lobos submediais e antes dos lobos mediais (Figura 6); gonóstilos (Figura 7) subtriangulares em vista lateral, com cerdas relativamente longas em sua margem ventral; lobos gonocoxais irregularmente arrendondados. 


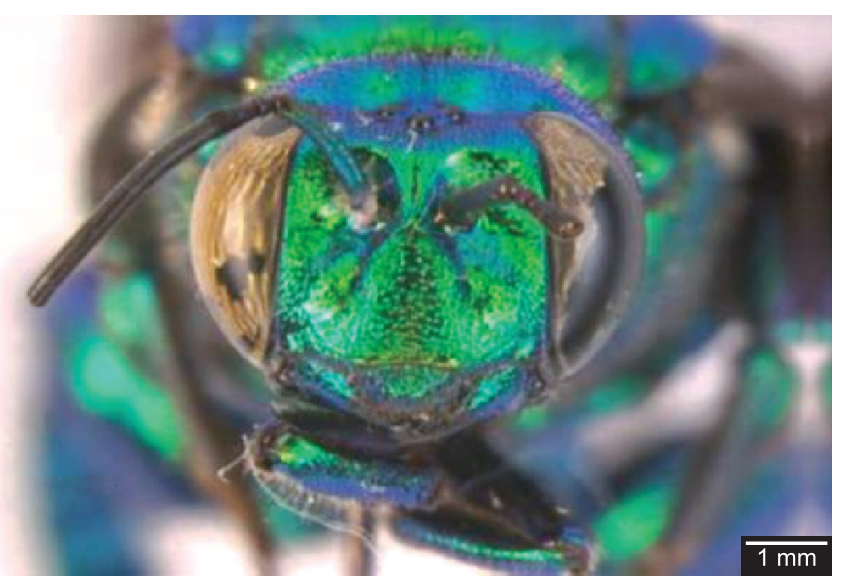

Figura 1. Exaerete kimseyae sp. n. vista frontal da cabeça.

Figure 1. Head of Exaerete kimseyae sp. n. in frontal view.

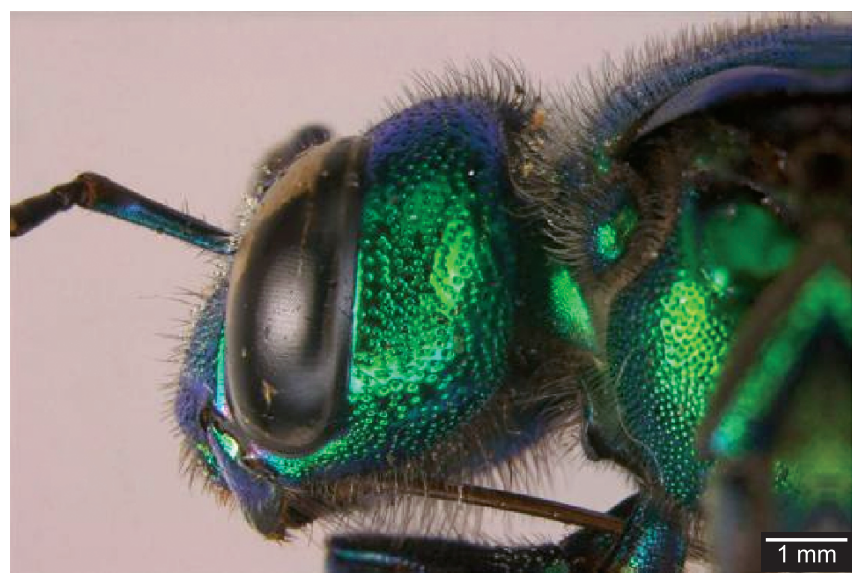

Figura 2. Exaerete kimseyae sp. n. vista lateral da cabeça.

Figure 2. Head of Exaerete kimseyae sp. n. in lateral view.

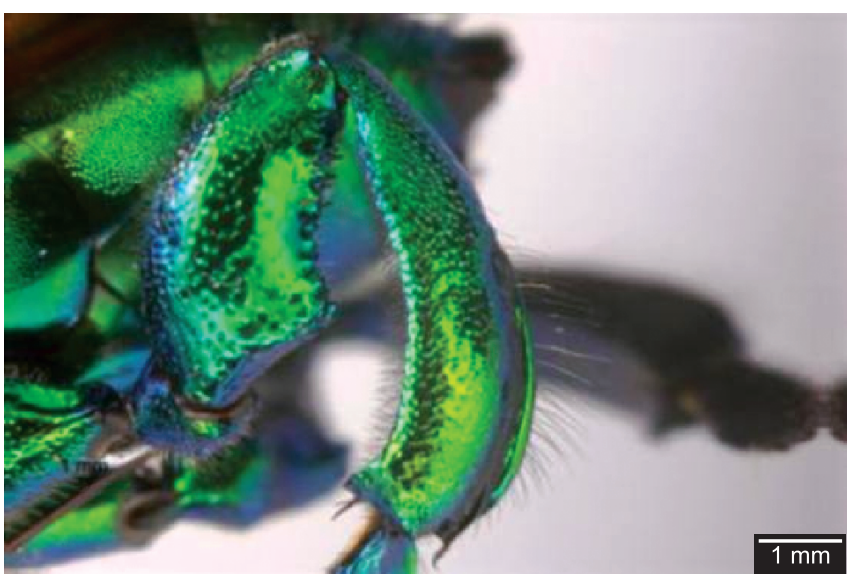

Figura 4. Exaerete kimseyae sp. n. vista lateral da perna posterior esquerda e suas projeções dentiformes.

Figura 4. Posterior leg of Exaerete kimseyae sp. n. in lateral view and its dental projection.

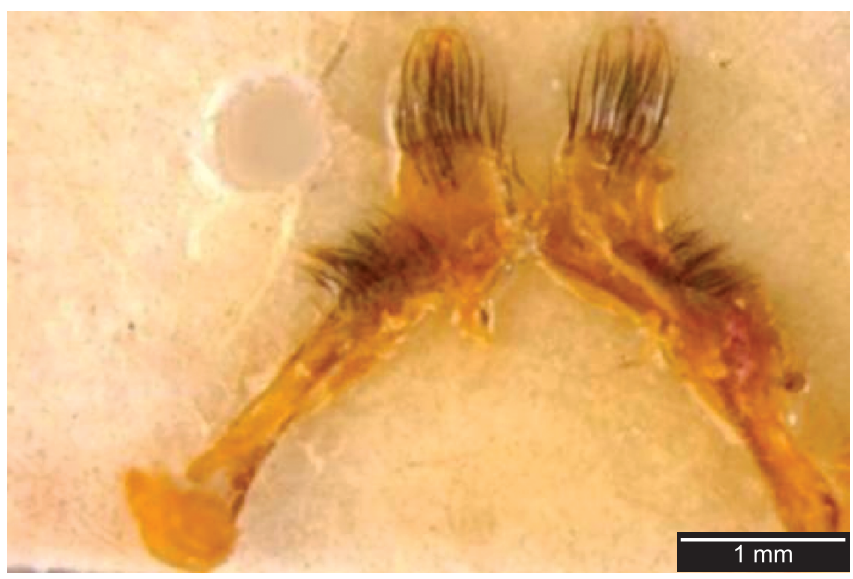

Figura 5. Exaerete kimseyae sp. n. vista dorsal do esterno VII.

Figure 5. Sternum VII of Exaerete kimseyae sp. n. in dorsal view.

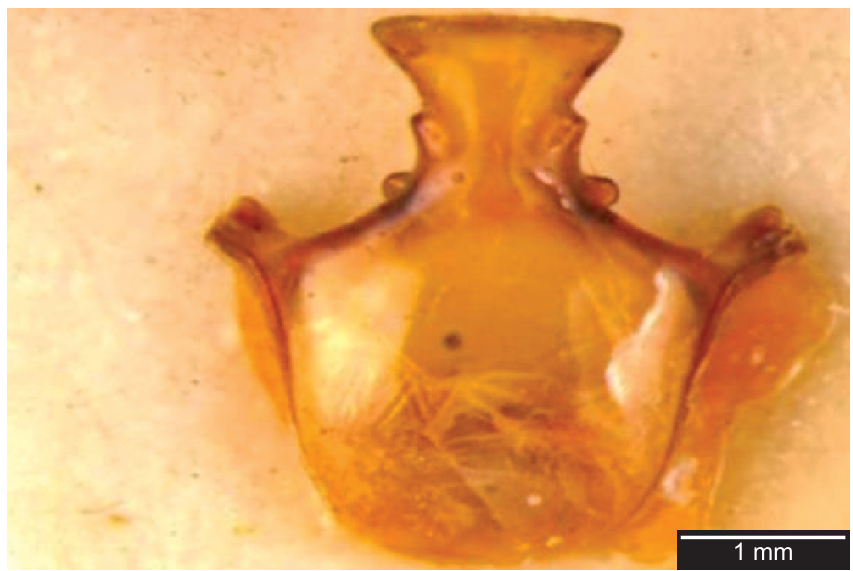

Figura 6. Exaerete kimseyae sp. n. vista dorsal do esterno VIII.

Figure 6. Sternum VIII of Exaerete kimseyae sp. n. in dorsal view. 




Figura 7. Exaerete kimseyae sp. n. vista lateral da cápsula genital.

Figure 7. Genital capsule of Exaerete kimseyae sp. n. in lateral view.

\section{Discussão}

Kimsey (1979) comenta que o grupo frontalis, constituído por E. frontalis (Guérin-Menéville, 1845), E. smaragdina (Guérin-Menéville, 1845) e E. trochanterica (Friese, 1910) possui palpos labiaistetrasegmentados; margem posterior do escutelo convexa; hipo-epímeros com um tubérculo bem desenvolvido; fenda tibial dos machos atingindo o ápice da tíbia e gonóstilos subtriangulares. De fato, os palpos labiais são bisegmentados nas duas primeiras espécies, a margem posterior do escutelo é reta na primeira e na terceira e o hipo-epímero possui um tubérculo bem desenvolvido apenas nas duas primeiras. Por outro lado, aquela autora comenta logo em seguida que $E$. trochanterica não é muito próxima às outras duas espécies e que talvez pudesse ser considerada representante de um terceiro grupo.

Entretanto, em sua chave, E. trochanterica sai junto com espécies do grupo dentata, constituído por E. dentata (Linnaeus, 1758) e E. azteca Moure, 1964, as quais possuem hipo-epímeros desprovidos de protuberância e o escutelo com margem posterior linear ou côncava, exceto para E. trochanterica [passo 1, segunda alternativa]. Porém, a figura 14 apresentada pela autora como sendo o escutelo de E. trochanterica, possui a margem posterior convexa, o que é confirmado mais adiante em sua discussão sobre essa espécie (Kimsey 1979). Na verdadeira E. trochanterica a referida margem é levemente côncava e com projeções dentiformes póstero-laterais, mais semelhantes a $E$. dentata. A mesma confusão acontece com a figura 24 relativa aos esternos VII, VIII e cápsula genital.

Ao que tudo indica, Kimsey desenhou essas estruturas tendo em mãos o exemplar do Panamá que corresponde a E. kimseyae sp. n., ao passo que seus outros desenhos (figuras 9, 19) tanto podem ser de E. trochanterica quanto de E. kimseyae sp. n.

\section{EXAERETE TROCHANTERICA (FRIESE, 1900)}

Chrysantheda trochanterica Friese, 1900. Entomol. Nachr. 26(5): 66. Holótipo fêmea: Brasil, Pará, Belém (ZMB; examinado).

Exaerete guaycuru Anjos-Silva \& Rebêlo, 2006. Zootaxa 1105: 27-35 (Sp. n., descr. orig., morf., tax.,). - Moure, Melo
\& Faria Jr., 2007: 254. In: J.S. Moure, D. Urban \& G.A.R. Melo (orgs.). Catalogue of Bees (Hymenoptera: Apoidea) in the Neotropical Region (Cat.). - Anjos-Silva, Engel \& Andena, 2007. Apidologie 38: (Fil., biogeo.). (examinado). Novo sinônimo.

Anjos-Silva \& Rebêlo (2006) não reconheceram que tinham em mãos um exemplar de E. trochanterica e o descreveram como E. guaycuru. Chegaram a essa conclusão com base no trabalho de Kimsey (1979), como explicitado acima. Aqui , E. guaycuru Anjos-Silva \& Rebêlo, 2006 é colocada como sinônimo-júnior de E. trochanterica, ao passo que suas figuras e descrição passam a servir agora para o reconhecimento de E. trochanterica.

Posteriormente, Anjos-Silva et al. (2007) colocaram E. lepeletieri como sinônimo de $E$. frontalis. Mencionam que a única e "insignificante" diferença entre elas estaria na margem apical do sétimo esterno metassomático, a qual possui forte lobo mediano em $E$. frontalis e relativamente fraco em E. lepeletieri. Não foram consideradas por eles, por exemplo, a ausência da protuberância na fronte, o escutelo com bordo posterior reto e a ausência de depressão na face interna dos fêmures posteriores, caracteres que distinguem nitidamente E. lepeletieri de E. frontalis. Tampouco foi considerado o fato da distribuição geográfica de E. lepeletieri ser restrita à bacia amazônica e a de E. frontalis ocorrer da América Central até o sul do Brasil.

\section{Agradecimentos}

A Lynn Siri Kimsey, Evandson dos Anjos-Silva e Frank Koch pelo empréstimo dos exemplares e tipos estudados.

\section{Referências Bibliográficas}

ANJOS-SILVA, E.J. \& REBÊLO, J.M.M. 2006. A new species of Exaerete Hoffmannsegg (Hymenoptera: Apidae: Euglossini) from Brazil. Zootaxa 1105:27-35.

ANJOS-SILVA, E.J., ENGEL, M.S. \& ANDENA, S.R. 2007. Phylogeny of the cleptoparasitic bee genus Exaerete (Hymenoptera: Apidae). Apidologie 38:1-7.

GARÓFALO, C.A. \& ROZEN-Jr., J.G. 2001. Parasitic Behavior of Exaerete smaragdina with descriptions of its mature oocyte and larval instars (Hymenoptera: Apidae: Euglossini). Am. Mus. Novit. 3349:1-26.

KIMSEY, L.S. 1979. An illustrated key to the genus Exaerete with description of male genitalia and biology (Hymenoptera: Apidae: Euglossini). J. Kansas Ent. Soc. 52(4):735-746.

MICHENER, C.D. 2000. The bees of the world. Johns Hopkins University Press, Baltimore.

MOURE, J.S. 1964. A key to the parasitic euglossine bees and a new species of Exaerete from Mexico (Hymenoptera: Apoidea). Rev. Biol. Trop. 12:15-18.

MOURE, J.S., MELO, G.A.R. \& FARIA-Jr., L.R.R. 2007. Euglossini Latreille, 1802. In Catalogue of Bees (Hymenoptera: Apoidea) in the Neotropical Region. (J.S. Moure, D. Urban \& G.A.R. Melo, orgs.). Sociedade Brasileira de Entomologia, Curitiba, p.214-55.

OLIVEIRA, M.L. \& NEMÉSIO, A. 2003. Exaerete lepeletieri (Hymenoptera: Apidae: Apini: Euglossina): a new cleptoparasitic bee from Amazonia. Lundiana 4(2): 117-120.

ROUBIK, D. \& HANSON, P.E. 2004. Abejas de orquídeas de la América tropical. Biología y guia de campo. INBio-Instituto Nacional de Biodiversidad. Santo Domingo de Heredia, Costa Rica. 